\title{
Hyperspectral Confocal Fluorescence Microscope: A New Look into the Cell
}

\author{
M. Cristina Pedroso*, Michael B. Sinclair**, Howland D. T. Jones**, and David M. Haaland** \\ * Monsanto Company, 800 N Lindbergh Blvd, U106N, St. Louis, MO 63117 \\ ** Sandia National Laboratories, Albuquerque, NM 87185-0895
}

The need for accurate quantification of in vivo cellular processes and more in-depth knowledge of organelle development and microstructure led Monsanto to search for non-commercial microscopes that could achieve those goals. Through a cooperative research and development agreement (CRADA) established between Monsanto and Sandia Corporation in August 2006, we built a new 3D-hyperspectral confocal fluorescence imaging system (Fig. 1), specifically designed to meet the analytical requirements of plant specimens. This instrument has the ability to follow many spectrally and spatially overlapping tags simultaneously and to discriminate them against autofluorescence or impurity emissions [1]. The 3D-hyperspectral images obtained are analyzed using Sandia proprietary Multivariate Curve Resolution (MCR) algorithms that can reveal emitting components with no a priori information, determine the emission spectrum of each component, and provide relative concentration maps of each component $[2,3]$.

Hyperspectral imaging of maize (Zea mays) leaf cross-sections followed by MCR analysis showed the presence of five pure spectral components in those samples (Fig. 2A). In contrast only two fluorescence components were detected by traditional filter-based confocal microscopes. Relative concentration maps revealed differences in chloroplast pigments between mesophyll and bundle sheath chloroplasts (Fig. 2B and Fig. 3). High spectral resolution and minimization of sources of noise during image processing also enabled the visualization of regions of grana in chloroplasts, and allowed the detection of the spatial distribution of chlorophylls a and b in chloroplasts (Fig. 4). Moreover, this study revealed the presence of autofluorescent components in the cytoplasm not detected by commercial microscopes. When compared with our two-photon Zeiss LSM510 META microscope, the hyperspectral microscope proved superior for: (1) experiments using dyes and/or fluorophores, namely, allowing the use of more labels per cell, lower concentrations of dyes, and detection of weak signals; (2) detection of fixation artifacts and false positives; (3) detection, identification, and quantification of autofluorescent cell components; and (4) separation of overlapping green fluorescence signals, such as green autofluorescence and green fluorescent protein (GFP) or Fluorescein Diacetate (FD).

Overall, this imaging diffraction-limited spatial resolution system has proven to offer a new look into the living cell, enabling the observation and quantitation of pure emission components never before achieved.

\section{References}

[1] M. B. Sinclair, et al. Applied Optics, 45 (2006) 6283.

[2] D.M. Haaland, et al. SPIE 4959 (2003) 55.

[3] H. D. T. Jones, et al. J. Chemometrics, 22 (2008) 482.

The support of Monsanto and Sandia management is gratefully acknowledged. 


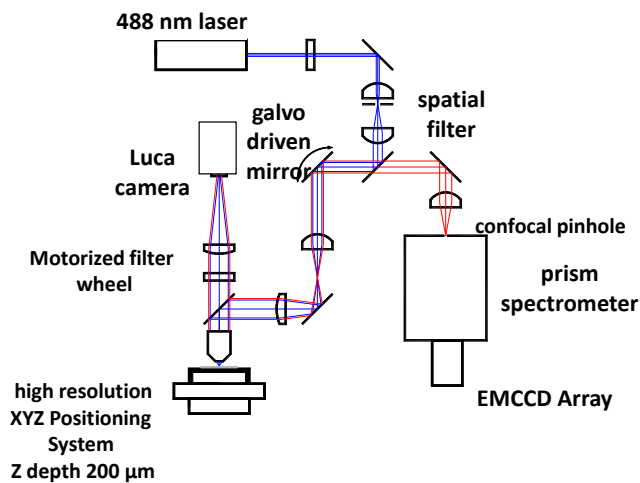

Figure 1- Optical layout of $1^{\text {st }}$ generation hyperspectral confocal fluorescence microscope.

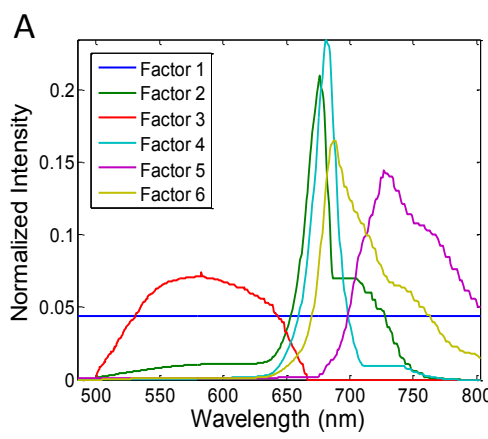

B

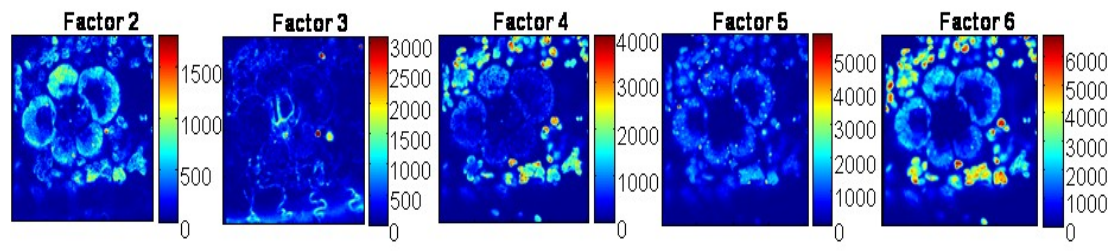

Figure 2. MCR analysis of hyperspectral images of corn leaf revealed the presence of five pure spectral components in those samples (A), and provided relative concentrations and the 3D spatial location of those pure components (B).

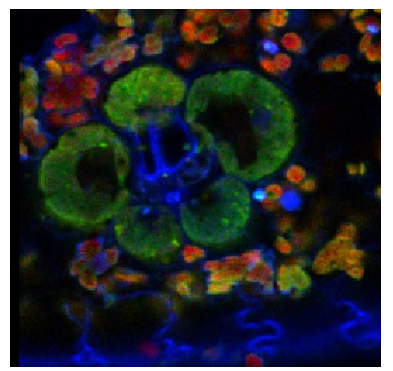

Figure 3. Hyperspectral microphotograph of a fresh, unstained corn leaf cross-section. The color of the fluorescence in this RGB image corresponds to the relative intensity of the colors in the spectral pure-component graph (Fig 2). Field of view $=100 \mu \mathrm{m}$.

Figure 4. Distribution of chlorophylls a (red component) and b (green) in a maize chloroplast.

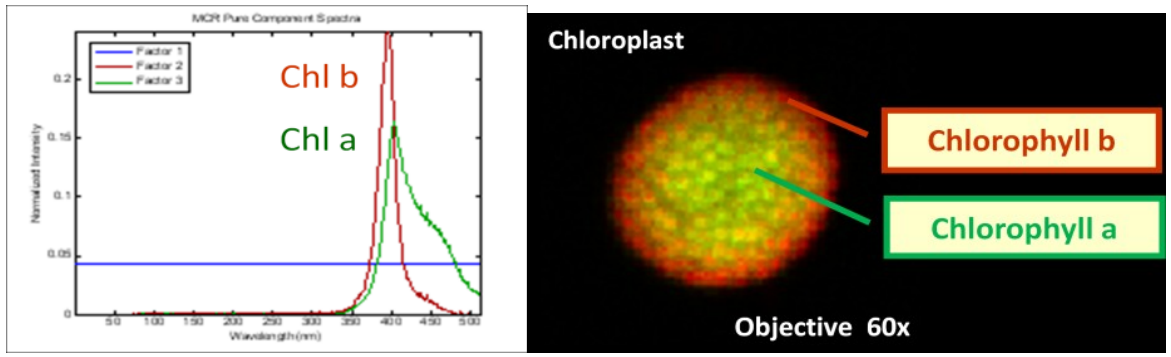

